\title{
Erratum to: Seroepidemiology of a second epidemic of hepatitis $E$ in a population that had recorded first epidemic 30 years before and has been under surveillance since then
}

\author{
Mehnaaz Sultan Khuroo • Mohammad Sultan Khuroo
}

Published online: 27 April 2010

(C) Asian Pacific Association for the Study of the Liver 2010

\section{Erratum to: Hepatol Int}

DOI 10.1007/s12072-009-9159-5

The author's proof corrections for Table 2 were only partially incorporated. The fully corrected table is reproduced here.

Table 2 Attack rate of hepatitis E infection (overall), icteric and anicteric hepatitis E infection rates in children and adults during 2007-2008 epidemic

\begin{tabular}{|c|c|c|c|c|c|}
\hline \multirow{2}{*}{$\begin{array}{l}\text { Age group } \\
\text { Number tested }\end{array}$} & \multirow{2}{*}{$\begin{array}{l}\text { Total } \\
N=638\end{array}$} & \multirow{2}{*}{$\begin{array}{l}\text { Children }(\leq 14 \text { years }) \\
N=330\end{array}$} & \multirow{2}{*}{$\begin{array}{l}\text { Adults ( }>14 \text { years) } \\
N=308\end{array}$} & \multicolumn{2}{|c|}{ Children versus adults } \\
\hline & & & & $P$ value & OR $(95 \% \mathrm{CI})$ \\
\hline HEV infection & $138(21.6 \%)$ & $78(23.6 \%)$ & $60(19.4 \%)$ & 0.21 & $1.27(0.86-1.80)$ \\
\hline Icteric HEV & $21(3.3 \%)$ & $6(1.8 \%)$ & $15(4.9 \%)$ & 0.03 & $2.76(1.06-7.02)$ \\
\hline Anicteric HEV & $117(18.3 \%)$ & $72(21.8 \%)$ & $45(14.6 \%)$ & 0.02 & $1.63(1.1-2.36)$ \\
\hline Icteric:anicteric ratio & $1: 5.6$ & $1: 12.0$ & $1: 3.0$ & 0.005 & - \\
\hline
\end{tabular}

The data show that attack rate of overall hepatitis E infection in children and adults did not differ. Adults more often presented with icteric disease, while children more often had anicteric infections

The online version of the original article can be found under doi:10.1007/s12072-009-9159-5.

Mehnaaz Sultan Khuroo ( $\square)$

Department of Pathology,

Sher-i-Kashmir Institute of Medical Sciences,

Soura 190 001, Kashmir, India

e-mail: mkhuroo@yahoo.com

Mohammad Sultan Khuroo $(\bowtie)$

Digestive Disease Centre, Dr. Khuroo's Medical Clinic,

Srinagar 190 001, Kashmir, India

e-mail: khuroo@yahoo.com 